\title{
Abwahl einer Schriftführerin nach Austritt aus ihrer Fraktion. Zum Urteil des Verfassungsgerichtshofs des Saarlandes vom 3. Dezember 2007 - Lv 12/07
}

\author{
Florian Edinger
}

Können Schriftführer abgewählt werden, zumal wenn sie - wie im Saarland - dem Parlamentspräsidium angehören? Diese Frage ist noch immer umstritten. Nunmehr hat der Verfassungsgerichtshof des Saarlandes eine solche Abwahl für zulässig erklärt.

\section{Sachverhalt}

Im Jahr 2004 zog Bündnis 90/Die Grünen mit drei Abgeordneten in den Landtag des Saarlandes ein. Ihre parlamentarische Geschäftsführerin, die Abgeordnete Barbara Spaniol, wurde vom Landtag zur Schriftführerin gewählt; damit war sie nach dem Gesetz über den Landtag des Saarlandes zugleich Mitglied des Landtagspräsidiums. Im Jahr 2007 trat Spaniol aus der Fraktion und der Partei Bündnis 90/Die Grünen aus und wechselte zur Partei Die Linke, die nicht im Landtag vertreten war; Spaniol wurde deshalb fraktionslos. Bündnis 90/Die Grünen behielt auch mit nur noch zwei Abgeordneten den Fraktionsstatus. ${ }^{1}$ Die Fraktion teilte dem Präsidenten des Landtags mit, sie ziehe die ausgetretene Abgeordnete aus allen Mitgliedschaften und Funktionen im Landtag zurück. Gleichzeitig nominierte sie eine andere Abgeordnete aus ihrer Fraktion als neue Schriftführerin. Der Landtag folgte dem Vorschlag und wählte das Fraktionsmitglied Claudia Willger-Lambert anstelle der Abgeordneten Spaniol zur Schriftführerin. ${ }^{2}$

Diese wandte sich an den Verfassungsgerichtshof mit dem Antrag, den Landtagsbeschluss für verfassungswidrig zu erklären. Da die Mitglieder des Präsidiums nach dem Gesetz über den Landtag (LtG) „für die Dauer der Wahlperiode“ gewählt würden, sei eine Abwahl unzulässig. Außerdem trug sie unter Berufung auf das „freie Abgeordnetenmandat"3 vor, das Amt des Schriftführers sei mit der Abgeordneten-Eigenschaft verknüpft und nicht mit der Zugehörigkeit zu einer bestimmten Fraktion. Sie beantragte eine einstweilige Anordnung mit dem Ziel, bis zur Entscheidung in der Hauptsache Mitglied des Präsidiums zu bleiben.

1 Zwei Abgeordnete sind die Mindestzahl für die Fraktionsbildung nach $₫ 1$ Abs. 2 des Fraktionsrechtsstellungsgesetzes (SFrReStG).

2 Vgl. Landtag des Saarlandes, 13. Wahlperiode, Pl. 13/41 vom 29. August 2007.

3 Art. 66 Abs. 2 S. 1 SVerf entspricht Art. 38 Abs. 1 S. 2 GG. 


\section{Die Entscheidung des Verfassungsgerichtshofs}

Der Verfassungsgerichtshof wies sowohl den Antrag auf eine einstweilige Anordnung ${ }^{4}$ als auch den Antrag in der Hauptsache ${ }^{5}$ zurück. Zum einen sei es nicht in der Verfassung geregelt, dass die Mitglieder des Präsidiums für die Dauer der Wahlperiode gewählt werden, sondern im Gesetz über den Landtag, das - eine saarländische Besonderheit - geschäftsordnungsrechtliche Regeln für das Parlament enthält. Unabhängig davon, ob sich aus einfachem Recht ein Verbot der Abwahl ergebe oder nicht, lasse sich aus der Verfassung kein allgemeiner Anspruch der Abgeordneten auf die Einhaltung einfachgesetzlicher Regelungen ableiten. Zum anderen enthalte die Verfassung kein Verbot, Präsidiumsmitglieder abzuwählen.

Soweit ist die Begründung des Gerichts kurz und präzise. Dann aber stellt es überraschenderweise die Frage, ob es eine „ungeschriebene Verfassungsnorm“ gebe, die die Abwahl eines Präsidiumsmitglieds verbiete - und vermeidet es anschließend, sich inhaltlich damit auseinanderzusetzen. Stattdessen argumentiert es formal: Die Antragstellerin hätte sich auf eine solche ungeschriebene Verfassungsnorm berufen müssen, habe das aber unterlassen.

Das Gericht prüft sodann, ob der verfassungsrechtliche Abgeordnetenstatus dem einzelnen Abgeordneten einen Anspruch darauf gibt, dass das Parlament seine Verfahrensvorschriften unterhalb der Verfassung ihm gegenüber einhält. Nur dann könne sich nämlich aus einer willkürlichen Anwendung der Bestimmung zur Wahl der Schriftführer in $\$ 33$ LtG auch eine Verletzung des verfassungsrechtlichen Anspruchs auf Gleichbehandlung der Abgeordneten ergeben, der aus dem Mandat folge (Art. 66 Abs. 2 Satz 1 LV).

Das Verfassungsgericht verneint dies: Der Anspruch auf Gleichbehandlung der Abgeordneten beziehe sich nur auf die organschaftlichen Mitwirkungsrechte an der politischen Willensbildung des Parlaments, wie das Rede-, Stimm- und Fragerecht, das parlamentsinterne Wahl- und Initiativrecht, das Recht der Fraktionsbildung sowie die Bemessung der Abgeordnetenentschädigung. ${ }^{6}$ Der Posten des Schriftführers sei hingegen eine Funktionsstelle, die gerade nicht alle Abgeordneten in gleicher Weise innehaben könnten. Aus der Mandatsgleichheit könne deshalb nicht eine bestimmte Ausgestaltung funktionsbedingter parlamentarischer Ämter abgeleitet werden, wie zum Beispiel die Unabrufbarkeit gewählter Präsidiumsmitglieder.

Das Gericht streift sodann die Frage, ob Fraktionen ihre Mitglieder aus Ausschüssen abberufen können. Es deutet an, dass dies zu bejahen sei, da Abgeordnete kein Recht hätten, einem bestimmten Ausschuss anzugehören. Letztlich lässt das Gericht jedoch auch diese Frage offen, weil sie für die Abwahl von Präsidiumsmitgliedern nichts hergebe, da das Präsidium an der parlamentarischen Beratung und Beschlussfassung, auf die sich die gleichen Teilhaberechte der Abgeordneten bezögen, nicht unmittelbar beteiligt sei.

4 VerfGH des Saarlandes, Lv 11/07 e.A., Beschluss vom 4. September 2007, www.verfassungsgerichtshof-saarland.de (26. Februar 2009); vgl. auch in: LKRZ, 2. Jg. (2008), H. 1, S. 26.

5 VerfGH des Saarlandes, Lv 12/07, Urteil vom 3. Dezember 2007, www.verfassungsgerichtshofsaarland.de (26. Februar 2009); vgl. auch in: LKRZ, 2. Jg. (2008), H. 3, S. 96.

6 Ähnlich die Rechtsprechung des Bundesverfassungsgerichts, vgl. BVerfGE 80, S. 188 (Wüppesahl-Urteil), BVerfGE 84, S. 304 und 96, S. 264 (Fraktions- und Gruppenstatus). 
Schließlich führe eine Abberufung aus dem Präsidium auch nicht zu mittelbaren oder faktischen Beeinträchtigungen des freien Mandats, die die Rechte der Abgeordneten verletzen könnten. So sei die Abberufung weder als Druckmittel geeignet noch benutzt worden, um die freie Entscheidung der Antragstellerin zu beeinflussen.

Im Übrigen habe der Landtag die einfachgesetzliche Vorschrift von $₫ 33 \mathrm{~S} .1 \mathrm{LtG}$ nicht verletzt. Nach dieser Vorschrift wählt der Landtag „für die Dauer der Wahlperiode“ seinen Präsidenten, die Vizepräsidenten und die Schriftführer ,unter Berücksichtigung der verschiedenen Fraktionen“. Die Formulierung „für die Dauer der Wahlperiode“ schließe eine Abwahl im Verlauf der Wahlperiode keineswegs aus. Das Gericht begründet dies zum Ersten historisch: Der Reichstag hatte die Formulierung in seine Geschäftsordnung aufgenommen, als er die vierwöchige Probezeit des Präsidenten mit anschließender Wahl für lediglich jeweils eine Sitzungs-(nicht: Wahl-)Periode abschaffte ${ }^{7}$; die Abwahl wollte er seinerzeit nicht regeln. Zum Zweiten kombinierten einige Bundesländer die Wahl für die Dauer der Wahlperiode mit Vorschriften über die Abwahl; auch dies zeige, dass eine Wahl für die Dauer der Wahlperiode die Abwahl keineswegs ausschließe. Dies gelte auch für $₫ 33$ S. 2 LtG. Und zum Dritten sehe Art. 70 Abs. 2 der Landesverfassung und ihm folgend $₫ 33$ Abs. 2 LtG vor, dass die Mitglieder des Präsidiums ,unter Berücksichtigung der verschiedenen Fraktionen" gewählt werden. Da die Antragstellerin aus der Fraktion ausgetreten sei, für die der Landtag sie in das Präsidium gewählt habe, habe der Landtag sie abberufen und durch ein anderes Mitglied der betreffenden Fraktion ersetzen dürfen. Denn die von Art. 70 Abs. 2 der Landesverfassung geforderte Berücksichtigung der Fraktionen bezwecke, dass Präsidium so zu besetzen, das die Zusammenarbeit der Fraktionen und damit die Arbeit des Landtags insgesamt erleichtert und gefördert werde. Dieser Zweck bestehe während der gesamten Dauer der Wahlperiode, zum Beispiel auch bei einer Nachwahl für ein aus dem Parlament ausgeschiedenes Präsidiumsmitglied, also nicht nur bei der Wahl des Präsidiums zu deren Beginn. Durch den Austritt der Antragstellerin aus ihrer Fraktion habe die Besetzung des Präsidiums nicht mehr den ursprünglichen Vorstellungen des Landtags von der Berücksichtigung der Fraktionen entsprochen. Bei der Besetzung des Präsidiums brauche der Landtag der Berücksichtigung einer fraktionslosen Abgeordneten nicht den Vorrang zu geben vor einer - wenn auch kleinen - Fraktion.

\section{Stellungnahme}

Der Entscheidung des Gerichts ist im Ergebnis zuzustimmen. Sie streift Themen, die in der Parlamentarismusforschung - und auch in dieser Zeitschrift ${ }^{8}$ - seit langem kontrovers diskutiert werden: Können Mitglieder eines Parlamentspräsidiums oder eines Ausschusses abberufen werden? Welchen Stellenwert haben Regeln über die Gremienbesetzung nach Fraktionsproporz? Allerdings gibt das Gericht nicht auf alle diese Fragen Antworten.

7 Unter Hinweis auf Florian Edinger, Wahl und Besetzung parlamentarischer Gremien, Berlin 1992, S. 171.

8 Zuletzt Sebastian Lovens, Der Bundestag zwischen Wahl und Entsendung zu seinem Präsidium, in: ZParl, 39. Jg. (2008), H. 1, S. 18 ff. 
Es geht im vorliegenden Fall nicht um die Abwahl des Parlamentspräsidenten oder seiner Stellvertreter, sondern um die Abwahl einer Schriftführerin. In den meisten Parlamenten ist das Amt der Schriftführer nicht mit nennenswerten Entscheidungsbefugnissen verbunden; ihre Funktion beschränkt sich in der Regel darauf, den Präsidenten bei der Sitzungsleitung zu unterstützen, indem sie die Rednerliste führen, die Redezeit überwachen und bei der Auszählung der Stimmen helfen. ${ }^{9}$ Im Landtag des Saarlandes ist das anders: Sie sind Mitglieder des Präsidiums. Damit fallen sie unter Artikel 70 Abs. 2 der Verfassung, den das Gericht als zentrale Norm für seine Entscheidung heranzieht: Der Landtag „wählt den Präsidenten, die Vizepräsidenten und die übrigen Mitglieder des Präsidiums unter Berücksichtigung der verschiedenen Fraktionen". Das ist mehr als Fraktionsproporz, denn die Norm verlangt die Berücksichtigung der Fraktionen selbst - nicht nur, wie in Artikel 77 Abs. 1 Satz 2, ihres Stärkeverhältnisses. ${ }^{10}$ Artikel 70 Abs. 2 lässt sich also durchaus so lesen, dass die Fraktionen - also alle - im Präsidium berücksichtigt werden müssen.

Dies erscheint auch durchaus sachgerecht, denn das Präsidium ${ }^{11}$ ist ein echtes Beschlussgremium mit bedeutenden innerorganisatorischen Kompetenzen: Es „beschließt über die inneren Angelegenheiten des Landtages, soweit sie nicht dem Präsidenten vorbehalten sind; insbesondere stellt das Präsidium den Entwurf des Haushaltsplanes für den Landtag fest und verfügt über die Verwendung der Räume im Landtagsgebäude. Die Beschlüsse werden mit einfacher Mehrheit gefasst. Bei Stimmengleichheit gibt die Stimme des Präsidenten den Ausschlag. "12

Im Ergebnis zu Recht geht das Gericht davon aus, dass diese Verfassungsbestimmung der Abwahl eines Präsidiumsmitglieds keinesfalls entgegensteht. ${ }^{13}$ Es gibt auch keine andere Verfassungsbestimmung, die dies verbietet, insbesondere kann die Antragstellerin aus ihrem Abgeordnetenmandat keinen Anspruch auf Verbleib im Präsidium ableiten. Erst recht existiert kein allgemeiner Verfassungsgrundsatz, der einer Abwahl eines Präsidiumsmitglieds entgegenstünde. Jedenfalls verbietet die Verfassung dies nicht, wenn sie über die Amtszeit der Präsidiumsmitglieder schweigt. ${ }^{14}$ Das Gericht kommt am Ende seiner Entscheidungsbegründung vielmehr zu dem Ergebnis, dass Art. 70 Abs. 2 der Verfassung den

9 Zum Bundestag vgl. Florian Edinger, a.a.O. (Fn. 7), S. 175. Siehe auch $₫ 36$ Abs. 1 LtG.

10 Art. 77 Abs. 1 S. 2 SVerf sieht vor, dass die Zusammensetzung der Ausschüsse „der Stärke der Fraktionen Rechnung zu tragen" hat.

11 Nicht zu verwechseln mit dem „erweiterten Präsidium“ nach $\$ 32 \mathrm{LtG}$, das die Funktion des Ältestenrats hat und keine Beschlüsse fasst.

$12 \$ 31 \mathrm{LtG}$.

13 Zur vergleichbaren Bestimmung des Art. 38 II LV NRW siehe Harald Hemmer, Der Präsident des Landtags NRW, Düsseldorf 2000, S. 64 f.; zu Art. 8 Verf ND vgl. Michael Köhler, Die Rechtsstellung der Parlamentspräsidenten in den Ländern, Berlin 2000, S. 44.

14 Der Niedersächsische Staatsgerichtshof war bereits 1962 der Ansicht, dass die Frage der Abwahl des Präsidenten nicht von der Verfassung geregelt werde, sondern eine rein geschäftsordnungsrechtliche Frage sei, StGH 1/62, Drs. IV, Nr. 912, S. 4760, Urteil vom 13. August 1962. Zu Art. 40 GG Hans H. Klein, in: Theodor Maunz / Günter Dürig (Hrsg.), Grundgesetz. Kommentar, Art. 40 Rn. 91; Florian Edinger, a.a.O. (Fn. 7), S. 170 ff.; Gerald Kretschmer, in: Bruno SchmidtBleibtreu / Franz Klein, Kommentar zum Grundgesetz, Art. 40 Rn. 38, Neuwied 2004; Hans D. Jarass / Bodo Pieroth, Grundgesetz für die Bundesrepublik Deutschland. Kommentar, Art. 40 Rn. 1, München 2007; Wolfgang Härth, Abwählbar oder nicht? Der Parlamentspräsident und sein Amt, in: ZParl, 16. Jg. (1985), H. 4, S. 490 - 495. Vgl. auch BVerwG 8 B 65.96 (Abberufung Kreistagspräsident), Beschluss vom 11. April 1996. 
Austausch der Antragstellerin gegen eine „grüne“ Schriftführerin sogar rechtfertigt, und zwar wegen des Gebots, die Fraktionen zu berücksichtigen. Dessen Zweck, die Zusammenarbeit im Landtag zu fördern, bestehe nämlich nicht nur zum Zeitpunkt der Wahl am Beginn einer Wahlperiode, sondern während ihrer gesamten Dauer.

Das Gericht hätte es durchaus dabei belassen können. ${ }^{15}$ Die Frage, ob Fraktionen Ausschussmitglieder abberufen können, ist für den vorliegenden Fall in der Tat irrelevant. Die Prüfung, ob der Landtag durch willkürliche Anwendung einfachen Rechts die Verfassung verletzt hat, ist allerdings vertretbar. Ansatzpunkt ist $\$ 33$ S. $1 \mathrm{LtG}$, der vorsieht, dass Präsidenten, Stellvertreter und Schriftführer für die Dauer der Wahlperiode gewählt werden. Dass auch diese Formulierung einer Abwahl nicht entgegensteht, begründet das Gericht ausführlich und zutreffend. Insofern kann von Willkür freilich keine Rede sein, zumal das verfassungsrechtliche Gebot der Berücksichtigung der Fraktionen die Abwahl rechtfertigt.

Wenn das Gericht sich schon mit unterverfassungsrechtlichen Normen befasst, hätte es an dieser Stelle allerdings nahegelegen zu prüfen, ob die Abwahl zulässig ist, auch wenn sie nicht ausdrücklich geregelt ist. Man kann hier formal argumentieren, die Abwahl sei ein actus contrarius, also das - ungeregelte - Gegenteil der Wahl, und hierfür hätten dieselben Regeln zu gelten wie für die Wahl. ${ }^{16}$ Für die Präsidenten und ihre Stellvertreter werden allerdings gewichtige inhaltliche Argumente dafür geltend gemacht, eine Abwahl nur unter bestimmten, ausdrücklich festgelegten Regeln zuzulassen. Insbesondere die Stellung der Präsidenten im Staat, ihre repräsentative Funktion und ihre unparteiische Amtsführung würden gefährdet, wenn sie jederzeit in einem rechtlich nicht näher ausgestalteten Verfahren abberufen werden könnten. ${ }^{17}$ Die Staatspraxis sieht das offenbar genauso: Die Abwahl von Parlamentspräsident und Stellvertreter ist mittlerweile in vielen Ländern ausdrücklich geregelt ${ }^{18}$; zum Teil ist sogar vorgesehen, dass ein Amtsinhaber beim Ausscheiden aus seiner Fraktion sein Amt automatisch verliert. ${ }^{19}$ Für die Schriftführer gelten die im Hinblick auf die Präsidenten und ihre Stellvertreter vorgebrachten Gründe für eine ausdrückliche Regelung zwar nicht in gleichem Maße. Als Mitglieder des Präsidiums sollten sie jedoch den übrigen Mitgliedern bei der Abwahl ebenso gleichgestellt sein wie bei der Wahl. Eine Abwahl ohne ausdrückliche Regelung mag zwar keine Willkür sein. De lege ferenda spricht allerdings viel für eine ausdrückliche Regelung der Abwahl oder des Amtsverlustes beim Ausscheiden aus der Fraktion. Ein entsprechender Hinweis des Gerichts wäre zum Abschluss der Begründung hilfreich gewesen.

15 Ähnlich wie der Niedersächsische Staatsgerichtshof, a.a.O. (Fn. 14).

16 Vgl. dazu Thomas Wilrich, Der Bundestagspräsident, in: DÖV, 55. Jg. (2002), H. 4, S. 152 ff. mit weiteren Nachweisen.

17 So insbesondere Michael Köhler, a.a.O. (Fn. 13), S. 45 ff.

18 In Bayern, Brandenburg, Mecklenburg-Vorpommern, Niedersachsen, Sachsen-Anhalt und Schleswig-Holstein in der Verfassung, in Nordrhein-Westfalen und Thüringen in der Geschäftsordnung.

$19 \$ 5$ Abs. 5 GOLT Niedersachsen, $\$ 3$ Abs. 7 GOLT Sachsen, $₫ 4$ Abs. 5 GOLT Sachsen-Anhalt, $\$ 2$ Abs. 4 GOLT Thüringen. 


\title{
4. Fazit: Bedarffür ausdrückliche Regelung
}

Die Entscheidung bestätigt die Tendenz, dass sich der Fraktionsproporz auch bei der Besetzung von gewählten Funktionsträgern durchsetzt ${ }^{20}$ - und zwar während der gesamten Wahlperiode. ${ }^{21}$ Es ist müßig, darüber zu spekulieren, ob die Entscheidung genauso ausgefallen wäre, wenn die Antragstellerin nicht zur Linken, sondern in eine etablierte Partei beziehungsweise Fraktion, vielleicht sogar in die Regierungsfraktion gewechselt wäre. Die Parlamente sollten Abwahl oder auch das Ausscheiden von Präsidiumsmitgliedern bei Fraktionswechsel jedenfalls ausdrücklich regeln.

20 Vgl. Florian Edinger, Fraktionen und Präsidium. Zum Anspruch jeder Fraktion auf Vertretung im Präsidium des Deutschen Bundestages, in: Recht und Politik, 31. Jg. (1995), H. 2, S. 77 - 84.

21 Zum Spannungsverhältnis Mehrheitswahl-Fraktionsproporz ausführlich Sebastian Lovens, a.a.O. (Fn. 8).

\section{Das Gedächtnis der Politik - Die Pressedokumentation des Deutschen Bundestages ${ }^{1}$}

\author{
Gerhard Deter
}

\section{Die historische Entwicklung im Überblick}

Die Pressedokumentation des Deutschen Bundestages weist inzwischen eine lange, komplexe, keineswegs aber geradlinig verlaufende Geschichte auf, die zu kennen unerlässlich ist, wenn man die gegenwärtige Situation dieser Institution verstehen und ihre Ressourcen umfassend nutzen will.

\subsection{Die Organisation}

Von 1949 bis 1957 wurde die Presseauswertung und -dokumentation, das selektive Sammeln, Verdichten, Analysieren, Ordnen, Archivieren und Vermitteln von Informationen also, als unselbständiger Bereich des Parlamentsarchivs geführt, wobei diese Arbeitseinheit zunächst als „Pressearchiv“, ab 1953 dann als „Presse- und Informationsstelle“ bezeichnet wurde. 1957 schließlich schuf die Bundestagsverwaltung unter dem Namen „Presseauswertung" ein eigenes Referat innerhalb ihrer Abteilung III, Wissenschaftlicher Dienst. Seit 1965 wurde die Pressedokumentation dann als selbständiges Referat der Unterabteilung „Presse- und Informationsstelle“ der Abteilung III geführt. Zu deren Aufgaben zählte nicht nur die so genannte „Inneninformation“, also die Presseauswertung, die Erstellung von

1 Der vorliegende Beitrag ist bereits in gekürzter Form erschienen, vgl. Gerhard Deter, Die Pressedokumentation des Deutschen Bundestages, in: Archivar, 61. Jg. (2008), H. 3, S. $254-261$. 